\title{
A Study of Improving Eighth Graders' Learning Deficiency in Algebra by Applying a Realistic Context Instructional Design
}

\author{
Yu-Liang Chang ${ }^{1} \&$ Yu-I Huang ${ }^{2}$ \\ ${ }^{1}$ Graduate Institute of Educational Administration and Policy Development, National Chiayi University, Chiayi, \\ Taiwan \\ ${ }^{2}$ Pi-Tou Junior High School, Changhua, Taiwan \\ Correspondence: Yu-Liang Chang, Graduate Institute of Educational Administration and Policy Development, \\ National Chiayi University, Chiayi, Taiwan. Tel: 886-919-515-826. E-mail: aldy.chang@msa.hinet.net
}

Received: October 8, 2012 Accepted: November 29, 2013 Online Published: December 3, 2013

doi:10.5539/ies.v7n1p1 URL: http://dx.doi.org/10.5539/ies.v7n1p1

\begin{abstract}
The intention of this study was to improve the learning deficiency in algebraic learning and to enhance Taiwanese middle students' learning achievement and interest in algebra. By using a grade skipping experimental design, the research team intended to find out an effective way to benefit these students' leaning in abstract algebraic concepts. Therefore, this study aimed to explore how the "realistic context" instructional design influenced 8th graders' performance on algebraic grade skipping learning of "linear programming". A quasi-experimental design with a post-test was employed in this study. Samples were selected purposely from thirty-six 8th graders of a junior high school as the Experimental Group, while seventy-nine 12th graders of a senior high school were chosen as the Control Group. Data were mainly gathered by the linear programming achievement test after executing the instruction. Statistical analyses were performed to answer the research question. Findings indicated that there was no significant difference between 8th graders (Experiment) and 12th graders (Control) on the performance of the linear programming achievement test. This result indicated that the instructional material with a realistic context design used in this study did help students to learn the abstract algebra effectively.
\end{abstract}

Keywords: algebra learning, realistic context, instructional design, eighth grader, linear programming

\section{Introduction}

\subsection{Background and Theoretical Framework}

In the highly civilized world of 21 st century, mathematical knowledge and abilities became basic requirements in our daily lives and careers (Lin, 2003; Polya, 1945; Romberg, 2001). In our daily lives, we actually used the most popular mathematic ideas and theories, not even recognizing that we were living with mathematics. The more one knew about mathematics, the broader one may develop in his career life (Stein, 1999). However, mathematic educators reviewed mathematical teaching materials used in the past and found that they were designed with an emphasis on mathematician and adult thinking approaches. In addition, the instruction emphasized heavily on abstract mathematical symbols and the training of calculation skills (Huang, 2003). The de-conceptualized, de-experienced thinking of the materials also ignored the cognitive principles and the processes of discovering the truth. Since mathematic teachers overvalued symbolic calculations and proofs as well as answers for mathematical questions, students may naturally sense that mathematics was disconnected with real-life situations; and then they may form the habit of insisting more on calculation skills for answers instead of on the process of thinking and reasoning. Thus, more and more students thought that "math was a boring symbolic game which concerned nothing about the real-life", or "math was so difficult to master (learn)" (Wu \& Ye, 2002; Zheng, 2003).

According to Piaget's (1970) theory of cognitive development, the junior-high students were at the right developmental period of "formal operational period", and they should be able to practice hypothesis and deduction with abstract symbols (Huang, 2001). Therefore, most of countries put the concept of written symbols into the mathematical curriculum at 7 th or 8 th grades. However, there were a large number of students facing problems in algebraic learning (Huang, 2001). A possible reason was because students' mathematical learning 
experience in the past (in the elementary level) was mainly about concrete practices of numbers and graphs. Consequently, when it came to more abstract algebraic content, if the instruction were not designed properly connected with their life experience and prior knowledge, the degree of learning difficulty would be then notably increased.

In recent years, our government endeavored to work on the educational innovations to enhance the civic quality and national competitive ability (Ministry of Education [MOE], Taiwan, 2000). The implementation of the Grade 1 9 Consecutive Curriculum was the core in this reform, where teachers could design curriculum and instruction based on their instructional belief and goal in order to match local conditions and students' personality and special needs (MOE, 2003). Based on the purpose of this reform, the research team applied the theory of Realistic Mathematics Education (RME) (Freudenthal, 1973; De Lange, 1995) to an instructional designing for eighth graders' learning in algebra. Both students' prior knowledge and experience were considered while designing this algebraic learning material, as well as how the content was closely related to their real-life situations.

RME was initiated by Freudenthal and his companies in the early 70'. According to the ideas of "mathematics as a human activity" and "mathematics must be connected to reality", Freudenthal (1973) developed the theory of RME. The theory of RME advocated that mathematics education should be based on students' cognitive development, and focused on the real life context. Thus, students could actively construct their own knowledge gradually with the contextual questions containing mathematical concepts, and then realize the mathematic relations and laws through experiences. Moreover, through interactions with classmates and teachers, students may gradually advance their thinking level, and further internalize the mathematical concepts. Besides, scholars in the United States progressed an experimental innovation on math curriculum (Mathematics in Context, MiC), which was similar to the concept of RME and greatly successful (Romberg, 2001). Except for providing an interesting and comfortable learning environment for learners to discover and learn (Freudenthal, 1973; De Lange, 1987), RME also emphasized that students should gradually mathematicalize and upgrade their study level through trials, interactions, and discussions. At the end, students were able to concretely handle the knowledge of formal and structural mathematics for effective implementations (Freudenthal, 1991; Treffers, 1987). Therefore, RME was correspondent to the requirements of modern mathematic education, which valued both processes and outcomes. In this study, the research team believed that, through a well-designed mathematical instruction based on RME, students could learn easily while facing the traditionally difficult algebraic unit. They would also be more confident and motivated in learning math because they are trained with methods and habits of active learning in a positive and interactive learning context. Finally, better learning achievements would be accomplished.

To proof that the mathematical instruction with a realistic context design based on RME was truly helpful for normal students in their algebraic learning, the research team chose an algebraic unit from the curriculum of "senior high schools", and then experimented it as a grade skipping learning process on 8th graders. Students were guided by the contextual questions within this instructional material, and then might perform self-construction and social-construction in the learning process. Their thinking and reasoning abilities might be cultivated and inspired. Further, their learning achievement on the algebraic unit would be promoted. After analyzing and comparing algebraic units among curricula of junior and senior high schools, "linear programming" in 12th grade was selected to be the experimental unit, which was highly practical while facing decisions in daily lives (Fang, 1993). The reason of using this "grade skipping" design was to prove that, "once we worked harder to create a better learning environment for our students; they could definitely obtain superior achievements". Thus, if the targeted students reached better mathematical achievements, mathematics teachers should think about how to improve their mathematical teaching in the classrooms.

\subsection{Purpose}

Accordingly, the intention of this study was to improve the learning deficiency in algebraic learning and to enhance Taiwanese middle students' achievement and learning interest in algebra. Through applying a grade skipping experimental design, it was intended to search out an effective way to advance these students' leaning in abstract algebraic concepts. Thus, the main purpose of this study was to explore eighth graders' achievement on algebraic grade skipping learning by using mathematical instructional material with a realistic context design. Besides, evidences regarding to what problems they faced while learning and their feelings and solutions were also collected as a reference. 


\section{Research Design}

\subsection{Method and Participants}

A quasi-experimental design was employed in this study. Samples were selected purposely from thirty-six 8th graders of a junior high school in the central part of Taiwan, as the Experimental Group (Experiment), while seventy-nine 12th graders of a senior high school were chosen as the Control Group (Control). The Experimental Group was taught by the mathematical instructional material in a realistic context design for exploring its influence on eighth graders' algebraic grade skipping learning. After the instruction, the post-test, the linear programming achievement test, was administered to both groups.

\subsection{Instructional Design}

The mathematical instructional material in a realistic context design used in this study was designed by the research team based on the theory of RME. The content in this instructional design was extracted from the Nan-I High-School Textbook. There were eight units in this material. The first and second units were designed for reviewing what students learned before (their prerequisite knowledge, linear inequalities with two variables and its graphic solution). The third, fourth, and fifth units introduced the main concept of "linear programming". By considering the local conditions around the targeted schools (i.e. it was a rural and agricultural environment; rice was the main product in that area), the content in the textbook was irrelevant to these students' life experience. Accordingly, a new version of the instructional material with a realistic context design, closely relevant to their daily lives, was designed to cope with the farming, the agricultural product, and its sale business. A series of the contextual questions were also developed in order to guide these students to learn the mathematical concepts. The sixth unit focused on the application of the knowledge on linear programming, which aimed to reinforce what they learned in previous units. Besides, this instructional material was implemented associated with the cooperative learning model. Thirty-six eighth graders in the experimental group were teamed up heterogeneously for group activities. There were six teams totally in the experimental group, six students for each team. Another 79 twelfth graders from a senior high school served as the control group, which implemented a traditional instruction with the original content of the textbook.

\subsection{Data Collection and Analysis}

Moreover, based on the instructional material used in the experimental group and the original textbook used in the control group, a linear programming achievement test was developed to examine these students' learning achievement. This fifty-minute test was sent to two professors, three high school math teachers, and two junior high school teachers for establishing the construct validity. The revised version was then administered to 52 twelfth graders of another high school in Changhua as the pilot test. According to the analysis of the pilot test, 20 questions were chosen based on the analysis of item difficulty index and item discrimination index, which included 5 multiple-choice, 6 fill-in, and 9 calculation questions. The average difficulty index was .65 and the average discrimination index was .45 . Table 1 showed the feature of these 20 items corresponding to Bloom's cognitive levels, while Table 2 for difficulty and discrimination indices of the instrument. 
Table 1. Items of the linear programming achievement test

\begin{tabular}{|c|c|c|c|c|c|c|c|}
\hline Content & Knowledg & mprehens & pplication & Analysis & Synthesis & aluation & $\begin{array}{l}\text { \# of } \\
\text { Items }\end{array}$ \\
\hline \multirow{3}{*}{$\begin{array}{c}\text { Linear Inequalities with Two } \\
\text { Variables }\end{array}$} & A: & A: & $\mathrm{A}: 2$ & A: 1 & A: & A: & \multirow{3}{*}{6} \\
\hline & B: & $\mathrm{B}: 1(1)$ & $B: 2$ & B: & $\mathrm{B}: 4(1)$ & B: & \\
\hline & C: & C: & C: & $\mathrm{C}: 1(3)$ & C: & C: & \\
\hline \multirow{3}{*}{ Linear Programming } & A: & A: & $\mathrm{A}: 4(1)$ & A:3 & $\mathrm{A}: 4(2)$ & A: & \multirow{3}{*}{8} \\
\hline & B: & $\mathrm{B}: 1(2)$ & $\mathrm{B}: 3$ & B: & B: & B: & \\
\hline & $\mathrm{C}:$ & C: & $\mathrm{C}: 1(2)$ & $\mathrm{C}: 2(2)$ & $\mathrm{C}: 2(3)$ & C: & \\
\hline \multirow{3}{*}{$\begin{array}{l}\text { Application of Linear } \\
\text { Programming }\end{array}$} & A: & A: & A: & A: & A: & A: & \multirow{3}{*}{6} \\
\hline & B: & B: & $\mathrm{B}: 4(2)$ & B: & B: & B: & \\
\hline & $\mathrm{C}: 1(4)$ & $\mathrm{C}: 1(1)$ & $\mathrm{C}: 1(5)$ & $\mathrm{C}: 2(1)$ & $\mathrm{C}: 2(4)$ & $\mathrm{C}:$ & \\
\hline$\#$ of items & 1 & 3 & 7 & 5 & 4 & 0 & 20 \\
\hline
\end{tabular}

Note: "A" is "multiple-choice question"; "B" is "fill-in question"; "C" is "calculation question".

Table 2. Indices of the linear programming achievement test

\begin{tabular}{cccc}
\hline Type of Questions \# of the question Difficulty Index Discrimination Index \\
\hline \multirow{4}{*}{ Multiple Choices } & 1 & .889 & .222 \\
& 2 & .861 & .278 \\
& 3 & .778 & .444 \\
& $4(1)$ & .889 & .222 \\
Fill-in & $1(2)$ & .684 & .479 \\
\hline \multirow{5}{*}{ Calculation } & $1(1)$ & .673 & .346 \\
& 2 & .738 & .364 \\
& 3 & .722 & .556 \\
& $4(1)$ & .712. & .423 \\
& $4(2)$ & .750 & .500 \\
& $1(1)$ & .667 & .667 \\
\hline \multirow{5}{*}{ Average } & $1(2)$ & .750 & .346 \\
& $1(3)$ & .639 & .500 \\
& $1(4)$ & .656 & .722 \\
& $1(5)$ & .430 & .534 \\
& $2(1)$ & .419 & .393 \\
& $2(2)$ & .545 & .756 \\
& $2(3)$ & .261 & .403 \\
& $2(4)$ & .259 & .406 \\
\hline
\end{tabular}

Data were mainly gathered by the linear programming achievement test after executing the instruction, associated with supplementary qualitative data collected from researchers' journals, students' learning records, and informal after-class interviews. Statistical analyses were performed to answer the research question. Quantitative and qualitative findings will be integrated to provide a thorough understanding and discussions of 
the algebraic grade skipping learning by using analysis in context strategy.

\section{Findings}

\subsection{Performance Comparison in Linear Programming Achievement}

In order to explore the difference between the learning achievement of Experiment and Control, a simple comparison was made on the average grade of each group. It showed that Experiment had the average score of 51.11 points, which was a little bit higher than Control, 50.28 points. Table 3 showed the summary of frequency and percentage in four different score levels for both groups. A chi-square significant test was carried out and the result was not significant, $\chi 2(3)=.285, p=.963>.05$. Further, the Shapiro-Wilk normality test was conducted, which was not significant for both groups, (Experiment) $p=.856>.05$ and (Control) $p=.321>.05$. In addition, the result of Levene's Test showed that $F(1,113)=.005, p=.945>.05$. These two results indicated that these data conformed with three basic assumptions: interclass independence, normality, and homogeneity of variances.

Table 3. Summary of frequency and percentage in four different score levels

\begin{tabular}{llllll}
\hline Group & $0-25 \mathrm{pts}$ & $26-50 \mathrm{pts}$ & $51-75 \mathrm{pts}$ & $76-100 \mathrm{pts}$ & Total \\
\hline Experiment & $1(2.8 \%)$ & $18(50.0 \%)$ & $15(41.7 \%)$ & $2(5.6 \%)$ & 36 \\
Control & $2(2.5 \%)$ & $38(48.1 \%)$ & $36(45.6 \%)$ & $3(3.8 \%)$ & 79 \\
Sub-total & $3(2.6 \%)$ & $56(48.7 \%)$ & $51(44.3 \%)$ & $5(4.3 \%)$ & 115 \\
\hline
\end{tabular}

Moreover, a $t$-test was conducted to examine the difference between students in Experiment and Control for their performances on the linear programming achievement test. The result was not significant, $t(113)=.280, p>.05$, see Table 4. This result clearly showed that, after receiving the experimental "algebraic grade skipping instruction (i.e. the instruction with a realistic context design)", there was no significant difference between Experiment, 36 eighth graders, and Control, 79 twelfth graders, on the performance of the linear programming achievement test. In fact, students in Experiment scored a little bit higher than those in Control (mean difference was .83). Consequently, the mathematical instructional material in a realistic context design did assist eighth graders to learn the abstract algebra (the content usually taught in 12th grade in Taiwan) effectively.

Table 4. Summary of the $t$-test

\begin{tabular}{ccccccc}
\hline Group & $N$ & $M$ & $S D$ & $t$ & $d f$ & $p$ \\
\hline Experiment & 36 & 51.11 & 15.039 & .280 & 113 & .780 \\
Control & 79 & 50.28 & 14.651 & & & \\
\hline
\end{tabular}

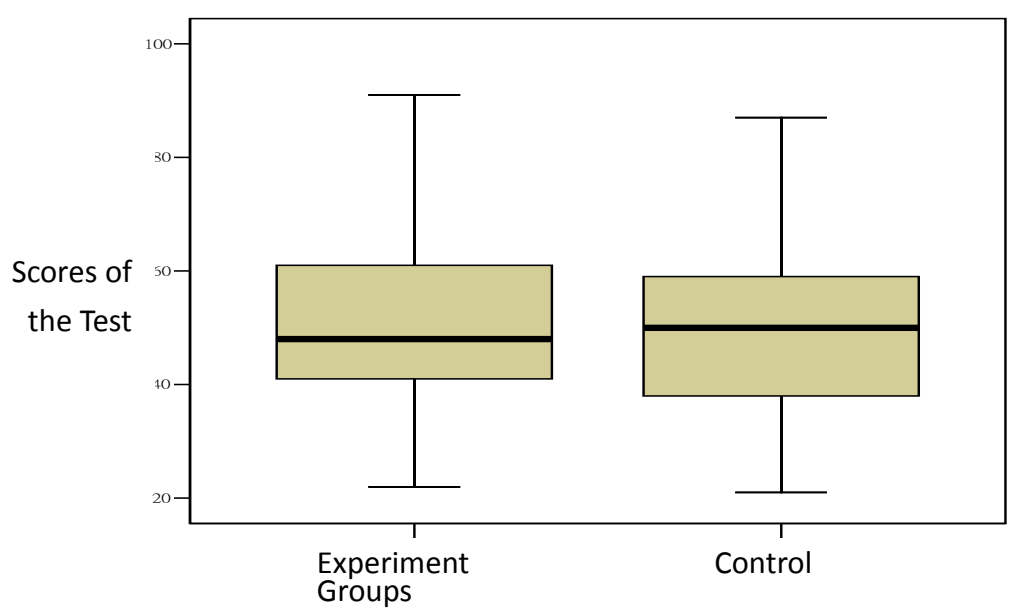

Figure 1. Distribution of the linear programming achievement test 


\subsection{Eighth Graders' Feelings While Facing Learning Problems and Their Solutions}

First, through the informal after-class interviews, over half of students in the experimental group thought that this instructional material with a realistic context design were more lifelike and easier to comprehend, which did help them better understand what they learned and how to apply what they learned into practice. They also thought it was a friendly, vivid, and interesting design. Besides, for the cooperative learning model, students in Experiment felt more freely to ask questions to classmates, express their own opinions, and listen to others within groups. During the process, they also felt the advantage of positive competitions within or among groups, which promoted them to be more active in learning the linear programming. Finally, they thought this kind of deign (the experimental instruction) was better than traditional ways, especially for those who were low-achievers. Therefore, they were expecting to experience more in their future learning processes.

Secondly, most of students in Experiment held positive opinions and evaluations toward this instructional material with a realistic context design. According to students' responses (in their learning records), "I do not know how to list the equation" was the most common problem they faced in the learning process. "I do not understand the meaning of some question, so I can not answer the following questions" and "the classmates are not cooperative" appeared to be other problems or complains. Moreover, most students tried to "discuss with classmates" to solve the problem faced. This was probably why "going to the teacher" became not so popular for their problem-solving strategy during the learning process of the last unit.

\section{Discussion and Conclusion}

First, the main problem that the learners (and the teacher) faced while using mathematical instructional material with a realistic context design is the context itself. As mentioned, a new version of the instructional material with a realistic context design, closely relevant to their daily lives, was designed to cope with the farming, the agricultural product, and its sale business. A series of the contextual questions were also developed in order to guide these students to learn the mathematical concepts (linear programing). This problem occurred when the targeted students reached this "agricultural" context. It was supposed that these students were familiar with the surrounding area where they lived and most of their parents' works were relevant to the agriculture and farm. However, it was ignored that these students were too young to have closed contacts with the agricultural business. Thus, the teacher actually took some time to introduce them the agricultural business. Consequently, it is suggested that, for designing this learning context, the context can be substituted by other issues that these students may deal with in their daily lives, such as taking the bus or the train. Or, the mathematics teacher can work closely with the social science teacher, who can introduce the surrounding area and the agricultural business to students in advance. In addition to this context problem, this realistic design was still considered as a successful design, especially for promoting students' discussions in the learning processes. Students worked in the small group could figure out a specific way of making their own statements. So, students could take turns to express their opinions about the question and the solution. Not only slower learners would have chances to learn from faster or smarter learners but also smarter learners could obtain opportunities to listen to others' opinions. This interactive exchange was truly beneficial for all students.

Secondly, as mentioned, the main purpose of this study was to improve the learning deficiency in algebraic learning and to enhance students' achievement in algebra. By using this grade skipping experimental design, the research team intended to find out an effective way to benefit these students' leaning in abstract algebraic concepts. The traditional algebraic course usually represented the most simple, formalized symbolic system and the calculation laws. Such a de-conceptualized and de-experienced design of teaching material was not able to connect students' knowledge and experience with real-life situations. Therefore, the degree of learning deficiency naturally increases (Huang, 2001). Besides, we found that eighth graders' algebraic learning abilities can be trained. Weng (2003) thinks that it is an infeasible thought to trade students' happy learning with simplification of teaching materials. Such a thought only weakens students' knowledge level, and even worse, limits the development of students' potential. In Romberg's (2001) study, learning achievement of high school students in U.S.A. and Netherlands were obviously advanced after receiving mathematical curriculum and instruction in realistic context. While in this study, eighth graders' achievement in the algebraic grade skipping learning of linear programming is greatly closed to the normal twelfth graders' achievement. Thus, what junior high students lack in algebraic learning may rather be the appropriate material and instruction than the ability itself. The only meaningful method for students shall be to design a well-planned material and instruction and practically apply them into practice. By doing so, their mathematical knowledge and abilities will be truly promoted, and the national competence will then be raised as well.

In summary, the instructional material with a realistic context design used in this study did help students to learn 
the abstract algebra effectively. Further, in this algebraic grade skipping learning, evidences indicated that students' potentials in learning could be promoted once an appropriate theory and corresponding instructional strategies were employed in the learning process. In fact, human brain's potentials in forming dispersions and connections are boundless ( $\mathrm{Wu} \&$ Chang, 2006). According to the recent findings of brain-based research, human brain is a seeker of models and meanings, which is characterized with self-invention, and will automatically learn numerous significant and useful knowledge and abilities in a learning context that is full of meaning and context (Wu \& Chang, 2006). Consequently, educators must take the responsibility of creating real-life learning environment, applying authentic learning activities, and encouraging interactions among all students in order to reach the ultimate goal of improving their learning process and equipping them proper knowledge and capability for their future lives.

\section{Endnote}

Actually, for the experimental group, a "mathematical learning attitude scale" was also administered as pre- and post-test for the purpose of understanding whether the instructional material with a realistic context design could influence these eighth graders' learning attitude or not. In fact, the targeted 8th graders' learning attitude towards mathematics was significantly better after receiving this realistic context instruction $(t=2.602, p<.01)$. In addition, it was also to explore how the two factors, "gender and ability", would influence upon their performance in algebraic grade skipping learning achievement (see Table 5 for the descriptive data). However, these results were excluded from this article.

Table 5. Descriptive Data of gender and mathematical ability of the 8th graders (Experiment)

\begin{tabular}{ccccc}
\hline 8th Gender & Ability & $M$ & $S D$ & $N$ \\
\hline \multirow{4}{*}{ Male } & Low & 46.17 & 13.06 & 6 \\
& Medium & 45.00 & 8.52 & 4 \\
& High & 65.43 & 16.31 & 7 \\
& Sub-total & 53.82 & 16.34 & 17 \\
\hline \multirow{4}{*}{ Female } & Low & 31.80 & 7.69 & 5 \\
& Medium & 52.78 & 10.58 & 9 \\
& High & 58.20 & 7.82 & 5 \\
& Sub-total & 48.68 & 13.76 & 19 \\
\hline \multirow{4}{*}{ Total } & Low & 39.64 & 12.86 & 11 \\
& Medium & 50.38 & 10.33 & 13 \\
& High & 62.42 & 13.46 & 12 \\
& Total & 51.11 & 15.04 & 36 \\
\hline
\end{tabular}

\section{References}

De Lange, J. (1987). Mathematics, insight, and meaning. Utrecht, Netherlands: OW\&OC.

De Lange, J. (1995). Assessment: No change without problems. In T. A. Romberg (Ed.), Reform in school mathematics and authentic assessment (pp. 87-172). New York: Sunny.

Fang, S. C. (1993). Linear programming. Mathematics Dissemination, 17(1), 1-12.

Freudenthal, H. (1973). Mathematics as educational task. Dordrecht, Netherlands: Reidel.

Freudenthal, H. (1991). Revisiting mathematics education. Dordrecht, Netherlands: Kluwer Academic.

Huang, W. X. (2003). How to do the educational reform? (Part Two) Permeate to the upper strata part. Journal of Educational Research, 106, 70-89.

Huang, X. M. (2001). Teaching idea and practice of life mathematics. Journal of Educational Research, 91, 63-73.

Lin, C. S. (2003). Mathematics education basic ideas. Retrieved May 4, 2005, from http://www.math.ntu.edu.tw/phpbb-2/edu/articles/article_03_05_06b.htm 
Ministry of Education. (2000). National elementary and middle schools nine years consistent curriculum temporary summary. Taipei, Taiwan: Author.

Ministry of Education. (2003). National elementary and middle schools nine years consistent curriculum summary. Taipei, Taiwan: Author.

Piaget, J. (1970). Piaget's theory. In P. H. Mussen (Ed.), Carmichael's manual of psychology (pp. 703-732). New York: Wiley.

Polya, G. (1945). How to solve it. New Jersey: Princeton University Press.

Romberg, T. A. (2001). Designing middle-school mathematics materials: Using problems set in context to help students progress from informal to formal mathematics reasoning. Madison, WI: University of Wisconsin-Madison, Wisconsin Center for Education Research.

Stein, S. K. (1999). Strength in number: Discovering the joy and power of mathematics in everyday life. Hoboken, NJ: Wiley.

Treffers, A. (1987). Three dimensions: A model of goal and theory description in mathematics education. Dordrecht, Netherlands: Kluwer Academic.

Weng, P. J. (2003). Discussed constructs the construction mathematics. Retrieved March 3, 2002, from http://www.wfc.edu.tw/ math/course.htm

Wu, B. L., \& Ye, C. H. (2002). Discussing the curriculum design of secondary mathematics statistics and probability based on the natural of potential and life mathematics. Journal of Education Research, 101, 90-104.

Wu, S. C., \& Chang, Y. L. (2006). Are you helping or obstructing your kids' brain development? Early Childhood Education Information Exchange, 182, 10-14.

Zheng, Y. H. (2003). Life and mathematics under the realistic mathematics view. Kang Hsuan Mathematics Teaching Communication, 1, 10-16.

\section{Copyrights}

Copyright for this article is retained by the author(s), with first publication rights granted to the journal.

This is an open-access article distributed under the terms and conditions of the Creative Commons Attribution license (http://creativecommons.org/licenses/by/3.0/). 